\title{
Non-monotonicity on a spatio-temporally defined cyclic task: evidence of two movement types?
}

\author{
S. Levy-Tzedek • Hermano Igo Krebs • \\ D. Song $\cdot$ N. Hogan $\cdot$ H. Poizner
}

Received: 5 June 2009/ Accepted: 22 January 2010/Published online: 19 February 2010

(c) The Author(s) 2010. This article is published with open access at Springerlink.com

\begin{abstract}
We tested 23 healthy participants who performed rhythmic horizontal movements of the elbow. The required amplitude and frequency ranges of the movements were specified to the participants using a closed shape on a phase-plane display, showing angular velocity versus angular position, such that participants had to continuously control both the speed and the displacement of their forearm. We found that the combined accuracy in velocity and position throughout the movement was not a monotonic function of movement speed. Our findings suggest that specific combinations of required movement frequency and amplitude give rise to two distinct types of movements: one of a more rhythmic nature, and the other of a more discrete nature.
\end{abstract}

Keywords Human motor control - Arm movement . Rhythmic $\cdot$ Cyclic · Discrete

\section{Introduction}

The relation between speed and accuracy has been extensively explored in the context of what has become known

S. Levy-Tzedek · H. I. Krebs $(\bowtie) \cdot$ N. Hogan

Massachusetts Institute of Technology, 77 Massachusetts,

Ave office 3-137, Cambridge, MA 02139, USA

e-mail: hikrebs@mit.edu

Present Address:

S. Levy-Tzedek

The Ben-Gurion University of the Negev,

Beersheba, Israel

D. Song $\cdot$ H. Poizner

University of California, San Diego, USA as the Fitts task, where task requirements are either spatially (Fitts 1954; Fitts and Peterson 1964) or both spatially and temporally (Schmidt et al. 1979; Wright and Meyer 1983) defined. This relation, which was found to be universally applicable in a wide variety of situations (albeit with some modifications, depending on the specific task requirements, e.g., Schmidt et al. 1979), using different end effectors, limbs and participant populations (see Table 1 in Plamondon and Alimi 1997) asserts that faster movements, as they allow less opportunity for online error correction, are less accurate than slower ones.

Speed-accuracy relationship

In a highly influential article in 1954, Fitts ${ }^{1}$ formulated a logarithmic relation between the duration and the required spatial precision of repetitive aimed upper limb movements, basing his theoretical model on information theory. A decade later, Fitts together with Peterson (1964) demonstrated that a logarithmic relation exists for discrete movements as well. This relationship was shown in later experiments to hold for different subject populations, end effectors and experimental paradigms (Wallace and Newell 1983; Meyer et al. 1988; Plamondon and Alimi 1997). Whereas some researchers anchored the logarithmic relationship in a different theoretical basis than that of Fitts (e.g., Crossman and Goodeve 1983), others found an altogether different trade-off relation; for example,

\footnotetext{
${ }^{1}$ Participants in this experiment were asked to use a stylus to alternatively strike two rectangular plates. The plates were of variable width, and the distance between them was varied as well, resulting in different levels of task difficulty. The level of difficulty was quantified as a function of the distance to the target, and the target's width, and referred to as the "index of difficulty" (ID). Movement time was measured as a function of the task's ID.
} 
Schmidt et al. (1979), described a linear speed-accuracy trade off using a modified Fitts paradigm, where both movement time and amplitude were controlled, and spatial error was the dependent variable. Wright and Meyer (1983) concluded that a logarithmic trade off was present when the task was spatially defined, and a linear relation came through when the task was temporally constrained.

There has been evidence of models' breakdown on tasks of a low ID (Beamish et al. 2006), of high frequency (e.g., Crossman and Goodeve 1983; Schmidt et al. 1979), of slow speeds (e.g., Schmidt et al. 1979), when comparing movements performed under different task conditions (e.g., discrete vs. rhythmic; Smits-Engelsman et al. 2002) or when performing two-handed movements of disparate difficulty (Kelso et al. 1979) but, to our knowledge, it has never been demonstrated that the relation may be non-monotonic.

\section{Discrete versus rhythmic movements}

Hogan and Sternad (2007) define discrete movements as ones preceded and succeeded by postures and occupying a non-negligible duration containing no posture, while rhythmic (or, synonymously, cyclic, repetitive or reciprocal) movements are commonly characterized by their periodicity. These authors make the point that there is a continuum between unambiguously rhythmic and unambiguously discrete movements, such that one may refer to the "degree of discreteness" and the "degree of rhythmicity" of a movement, rather than necessarily classify it as one or the other (see also Bootsma et al. 2004).

Whereas most researchers studying the speed-accuracy trade off focused on tasks of a discrete nature, some studied and compared both discrete and repetitive movements (Fitts 1954; Fitts and Peterson 1964; Crossman and Goodeve 1983; Schmidt et al. 1979; Guiard 1997; SmitsEngelsman et al. 2002, Buchanan et al. 2003). Their findings were not always in agreement. Fitts and Peterson (1964) concluded that both types of movement follow the same type of behavior, later known as Fitts' Law, with different slopes. Fitts' law describes a dependence between the time to complete a movement and the distance and size of the target (or the task's ID). It is interesting to note that Fitts and Peterson found cyclic movements took longer to complete than discrete movements of the same ID (Fitts and Peterson 1964). Crossman and Goodeve (1983) and Guiard (1997) also found Fitts' Law to apply to both types of movement, at least approximately.

In contrast, Schmidt et al. (1979) found that when movements are temporally constrained, while in discreteaiming tasks the endpoint error is directly related to the amplitude and inversely related to the movement time, for reciprocal movements, the endpoint error is directly related to the amplitude and independent of the movement time.
Smits-Engelsman et al. (2002) demonstrated that, contrary to Fitts and Peterson's (1964) results, when participants performed the same task in a discrete, and in a cyclic manner, for the same ID, the latter allows participants to reach twice the speed of the former. They posited that had cyclic movements been but a concatenation of discrete ones, there should have been little difference in the performance between the two. Their results are in line with the view that discrete and cyclic movements are governed by different control principles. Further support for the idea that cyclic and discrete movements are separately controlled comes from a brain-imaging study (Schaal et al. 2004), as well as from theoretical considerations (Guiard 1993).

Finally, Buchanan and colleagues performed a series of studies exploring the use of two different units of action, rhythmic and discrete, both separately and within the same movement, and found that as participants approach targets of higher ID, their movements become more discrete in nature, while they are more harmonic in nature when approaching a target of low ID (Buchanan et al. 2003, 2004, 2006). As Guiard pointed out earlier, such a correlation between harmonicity and task ID may in fact be a secondary effect, and the result of the slowing down of movement as one reaches to a target of higher ID, as predicted by Fitts' law (Guiard 1997).

In the traditional paradigm, participants either perform the task as fast as possible (e.g., Fitts 1954), or at a high frequency (movement time $=180-500 \mathrm{~ms}$, Schmidt et al. 1979; Wright and Meyer 1983), and accuracy is measured at the endpoints of the movement only.

We hypothesized that under further modification to the traditional paradigm, continuously constraining the movement both spatially and temporally, this relationship would be maintained. That is, accuracy would diminish as speed would increase.

Participants were asked to perform horizontal reciprocal flexion/extension movements with their forearm. The experimental paradigm was designed such that timing cues were not explicit, but rather, timing was implicitly dictated by a closed shape on a phase-plane display (where speed is plotted vs. position). The phase plane afforded a way to display target amplitude and frequency of movement to participants without giving them any explicit timing cues (e.g., via a metronome). It is widely used to study dynamical systems, especially those that typically underlie the production of rhythmic behavior (e.g., limit-cycle oscillators; Kelso and Tuller 1984). A key feature of the phase plane is that it "suppresses time"; that is, a phaseplane plot fully characterizes the dynamics of a onedimensional oscillator yet does not explicitly represent time. Therefore, by using a phase-plane display we were able to give precise instructions about target amplitude and 
frequency, while minimizing requirements for processing explicit timing information. In order to study which characteristics of the movement change based on visual feedback, we included in each experimental block a few "blind" trials, in which the participants did not get visual feedback on their movements.

Unlike the traditional paradigms used in studies of the speed-accuracy trade off, in our task, the closed shape displayed on the phase plane specified both the allowed speed ranges (slow, intermediate or fast), and the allowed movement amplitude. As such, accuracy is not required only at the endpoints, but rather along the entire path. That is, the task required spatio-temporal accuracy, as opposed to strictly spatial accuracy.

To our knowledge, the relation between speed and accuracy in a task that requires co-modulation of speed and position throughout the task, as opposed to tasks that are either spatially or temporally defined, has not been explored.

\section{Methods}

\section{Participants}

Twenty-three healthy adult participants without any known neurological disorders or tremor were tested using their dominant hand in this experiment (age, $52.7 \pm 22.3$ years; range 22-81 years; 10 females; 13 males). All participants gave their informed consent to participate. The protocol was approved by the Institutional Review Boards of the Massachusetts Institute of Technology and of the University of California, San Diego.

\section{Equipment}

The equipment used for this experiment consisted of a modified version of the elbow-angle measurement device described in Doeringer and Hogan 1998. A forearm support, consisting of a commercially available wrist splint (Futuro splint wrist brace) strapped to a flat aluminum plate atop a lightweight aluminum tube, was hinged via precision ball bearings to a stationary support, mounted on a table in front of the seated participant. The forearm support was deliberately designed to be as light as possible, to minimize its effect on the natural behavior of the limb. Its moment of inertia was $\sim 0.0056 \mathrm{~kg} \mathrm{~m}^{2}$, an order of magnitude less than the mean value of the participants' forearm moment of inertia, $\sim 0.075 \mathrm{~kg} \mathrm{~m}^{2}$.

The forearm support was connected to the shaft of a rotary incremental encoder (Gurley Precision Instruments Model \# R119) with a position resolution of 0.0003 radians per count. The encoder was in turn connected to a counter card inside a computer running the real-time Linux operating system. Using this angle sensor, we were able to display both the position and the velocity of the elbow directly to the participant in real time. The computer controlled the recording of the data, as well as the display, which was a 17-in. monitor, positioned $\sim 80 \mathrm{~cm}$ from a participant's eyes. Data were recorded at $200 \mathrm{~Hz}$. A large, opaque plastic cover was placed parallel to the table, and above the apparatus, such that during the experiment, the participant's forearm was occluded from view (see Fig. 1).

\section{Protocol}

Participants were presented with a display of the phase plane of their forearm motion; the horizontal axis displayed angular position and the vertical axis displayed angular velocity. The target forearm behavior was indicated by a region of the phase plane; this region was a doughnut shape formed from two ellipses displayed on the screen. Each ellipse corresponds to a sinusoidal motion about the elbow, with the nonzero width of the doughnut shape allowing for a range of amplitudes and frequencies. The protocol consisted of 3 blocks of 20 trials each. The three blocks were differentiated by the shapes of their target regions; the shape displayed was either (1) a tall thin region (fast,

(a)

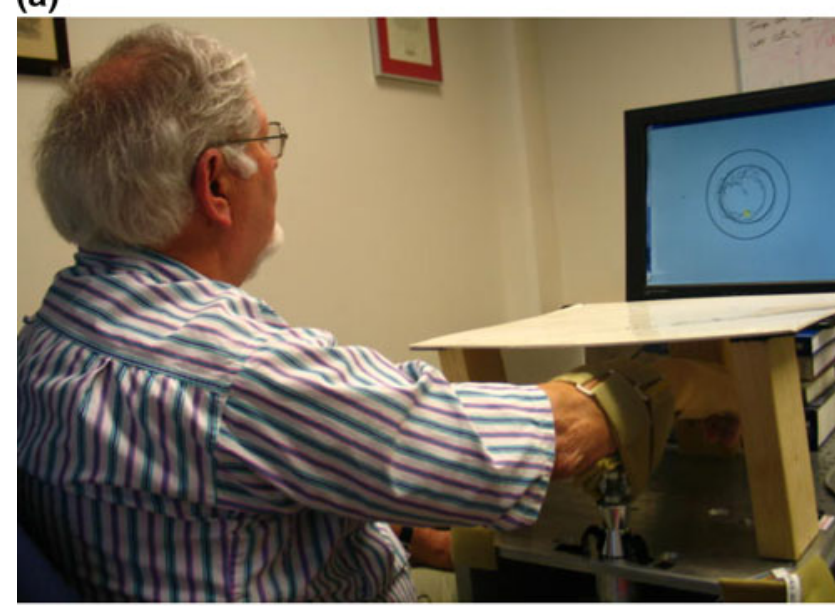

(b)

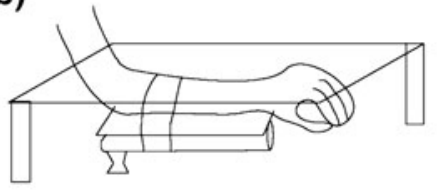

(c)

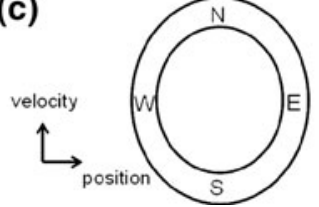

Fig. 1 The experimental setup. a The forearm is strapped to the angle-measuring device, and is occluded from the participant's view by an opaque cover. The participant coordinates simultaneous modulation of both speed and position to control a cursor displayed on a phase plane. b An illustration of the arm's position below the cover. c An example of the phase-plane display, with the four quadrants $(N, S, E, W)$ marked in their respective positions 
small-amplitude sinusoid), (2) a circular region, or (3) a wide region (slow, large-amplitude sinusoid; see Table 1 and Fig. 2). We kept the same dependence between the arm state variables (angular position and velocity) and the display for all blocks because we wanted to minimize unnecessary relearning of the relationship. That is, the same conversion factors were used between the arm state variables and the display in all the blocks. The order of presentation was altered and balanced across participants, such that approximately half of the participants were tested first on the fast block and approximately half were tested first on the slow one; the second block of trials always consisted of the medium-speed condition. Before each block of 20 trials, participants were allowed to practice the movement until they felt comfortable with the task, which usually took about four 40-s practice trials. Each test trial lasted for $20 \mathrm{~s}$. For each block of 20 trials, 5 of them (the second, the last, and three randomly selected) were blind; during these trials, participants could see the doughnutshaped target region, but not the trace corresponding to their own forearm motion. The instructions to the participants were as follows:

On the screen in front of you, you will see a cursor whose vertical position will depend on your elbow velocity, and whose horizontal position will depend on your elbow position. We ask that you move your elbow back and forth in cyclic movements (demonstrate) so that the cursor stays within the doughnut shape displayed on the screen. On some trials, the cursor will not be visible; you won't be able to see the trace of your movement on the screen. In those trials, continue to try and move within the guidelines even though you cannot see the trace (demonstrate).

Data analysis

Data were analyzed using MATLAB $^{\circledR}$ (7.0.1, The MathWorks, Natick, MA). Trend was removed from the position data, so as to reduce the effects of drift. This was achieved

Table 1 Frequency and amplitude values for the center of each of the three ellipses, as well as the allowed ranges for those parameters

\begin{tabular}{|c|c|c|c|c|c|c|}
\hline \multirow[t]{2}{*}{ Ellipse } & \multicolumn{3}{|c|}{ Frequency $(\mathrm{Hz})$} & \multicolumn{3}{|c|}{ Amplitude (degrees) } \\
\hline & Center & Range & Performed (mean $\pm \mathrm{SE}$ ) & Center & Range & Performed (mean $\pm \mathrm{SE})$ \\
\hline Tall (fast) & 2.3 & $1.3-5.5$ & $1.9 \pm 0.07$ & 6.7 & $3.3-10.2$ & $7.9 \pm 0.31$ \\
\hline Round (medium) & 0.55 & $0.36-0.85$ & $0.72 \pm 0.02$ & 16.3 & $12.9-19.8$ & $14.8 \pm 0.23$ \\
\hline Wide (slow) & 0.16 & $0.07-0.3$ & $0.20 \pm 0.007$ & 24.6 & $20.6-28.6$ & $22.9 \pm 0.23$ \\
\hline
\end{tabular}

Center frequency values correspond to the frequency of a movement trace along the center of each doughnut. Amplitude range values refer to the angular distance between the two inner (low value) and the two outer (high value) edges of each doughnut. Performed values are the actual values of frequency and amplitude at which participants executed the task (vision trials)

Fig. 2 Left column phase-plane trajectories from one participant in the slow, medium and fast vision blocks. $Y$ axis angular velocity $(\mathrm{rad} / \mathrm{s})$, $x$ axis position (rad). Right column velocity traces from the same participant in the slow, medium and fast blocks. $Y$ axis angular velocity $(\mathrm{rad} / \mathrm{s}), x$ axis time (s). Flexion $(F L X)$ and extension (EXT) directions are indicated on the top two plots
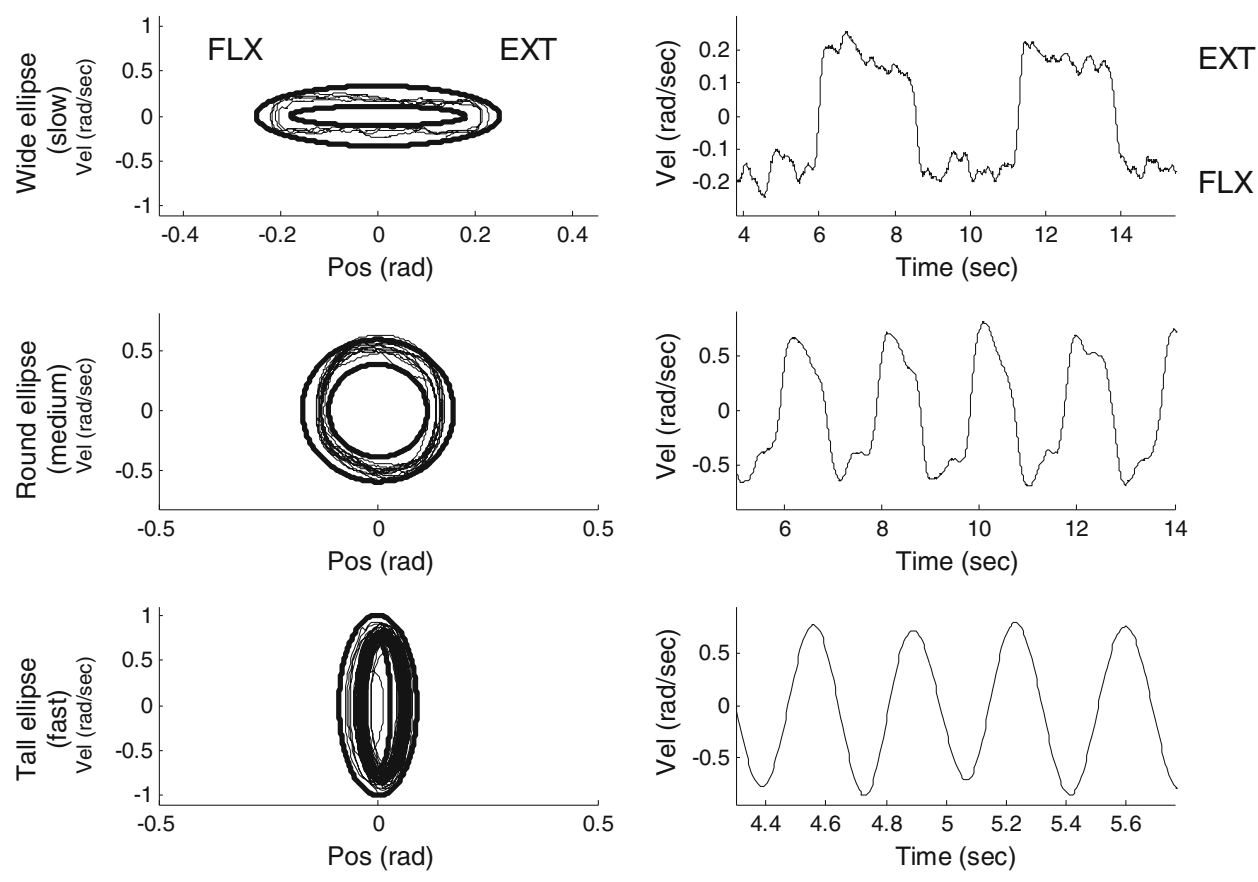
by removing the best straight-line fit from the angular position data. Position and each of its three derivatives, were filtered using a zero-phase (bidirectional) digital filtering with a first order Butterworth filter (bidirectional filtering doubles the filter order) with a cutoff frequency of $20 \mathrm{~Hz}$. Velocity was calculated as the difference between every two consecutive points in the filtered position record, multiplied by the sampling frequency and then filtered as described above. In a similar fashion, acceleration and jerk were calculated.

Some of the metrics described below were applied to complete movement cycles, whereas others were applied to each phase-plane quadrant separately. We divided the movement trace from each trial into four quadrants: around positive and negative target peak velocity ( $N$ and $S$, respectively; centered at the forearm's neutral position, at zero crossings in the position trace) and around movement reversals: from extension to flexion and from flexion to extension ( $E$ and $W$, respectively; see Fig. 1).

\section{Accuracy}

Each 20-s trial was given a numerical score that represented the percent of the total trial time that was spent inside the target zone on the phase plane. This procedure was repeated separately for each quadrant in the phase plane, as defined above.

\section{Variability in phase-plane path}

Movement traces from each phase-plane quadrant in each trial were resampled to a uniform length. The edges of the resultant vectors were removed, to avoid edge-effects due to resampling. The values from each quadrant were then normalized by the maximum value in that quadrant, and the variability in the path was calculated by taking the standard deviation of the path across the repetitions within the given quadrant.

\section{Acceleration time as a percent of half-cycle time}

The movement trace from each trial was segmented into half cycles (flexion or extension of the elbow), defined by zero crossings in the velocity trace. In each half cycle, the time to peak velocity was calculated, and divided by the total halfcycle time. A smaller proportion of movement time spent prior to reaching peak velocity has been interpreted as indicative of a longer proportion of movement time spent visually directing the movement (Ricker et al. 1999).

\section{Smoothness}

The smoothest rhythmic movement can be defined using a "mean squared jerk" (MSJ) measure (Nelson 1983).
Accordingly, to evaluate smoothness in each trial, we calculated the average of the rate of change of acceleration (jerk) squared, and divided it by the mean-squared jerk of a corresponding maximally smooth rhythmic movement (Hogan and Sternad 2007), to obtain the unitless meansquared jerk ratio (MSJR):

MSJ $=\frac{1}{t_{2}-t_{1}} \int_{1}^{2} \frac{1}{2}\left|\frac{\mathrm{d}^{3} x}{\mathrm{~d} t^{3}}\right|^{2} \mathrm{~d} t$

$\operatorname{MSJR}=\frac{\text { MSJ }_{\text {movement }}}{\text { MSJ }_{\mathrm{ms}}}$

where $\mathrm{MSJ}_{\mathrm{ms}}$ is the MSJ of the corresponding maximally smooth movement.

As described in appendix II of Hogan and Sternad (2007), the minimum-MSJ movement is strictly periodic and essentially sinusoidal. Accordingly, we used a sinusoid of the same duration, amplitude and number of peaks as the corresponding maximally smooth movement. Zero crossings in the velocity data were used to define peaks in the position data. Data points before the first and after the last velocity peak were discarded, so that a direct comparison can be made with the corresponding sinusoid. A ratio value approaching unity would indicate a mean-squared jerk value comparable to that of a maximally smooth movement, whereas a value much greater than one would imply the movement is highly fragmented.

\section{Frequency}

The average frequency of movement in each trial was estimated by calculating the reciprocal of twice the average peak-trough horizontal distance in the position record.

\section{Harmonicity}

Harmonicity of the movement is determined by the features of the acceleration trace (single or multi-peaked, with or without zero crossings) around movement reversals, and provides a measure of the harmonic or inharmonic nature of the movement (Guiard 1993). For every movement half cycle, between two zero crossings in the position record, the unitless harmonicity index was calculated as follows: when a single peak in acceleration occurred in the half cycle, the harmonicity value was set to one; when an inflection occurred in the half-cycle acceleration trace, movement harmonicity was computed as the ratio of the minimum to the maximum absolute value of the acceleration within the given half cycle; finally, if the acceleration trace within the half cycle changed its sign, the harmonicity value was set to zero (Guiard 1993; Buchanan et al. 2006). Harmonicity values were then averaged across the entirety 
of each 20-s trial. A harmonicity value approaching one indicates a highly harmonic movement, whereas a value approaching zero implies mechanical energy is dissipated in the vicinity of movement reversal (Buchanan et al. 2006).

\section{Posture}

A posture can be defined by a non-negligible duration in which only negligible movement occurs, and, if found in a series of reciprocal movements, may indicate the movements are of a discrete nature (Hogan and Sternad 2007). Around every movement reversal in each trial, the acceleration trace was scanned for values approaching zero, that is, below a certain threshold $\left(\varepsilon=0.1 \mathrm{rad} / \mathrm{s}^{2}\right)$, which were maintained for a duration greater than $\delta=6 \mathrm{~ms}$. If this condition was met, it was determined that posture occurred at this movement reversal. The percent of movement reversals in each trial that contained postures was then calculated.

\section{Statistical analysis}

Unless otherwise noted, a non-parametric paired test, the sign test, was used to test the significance of differences between data sets. This non-parametric test was chosen to eliminate the need for assumptions regarding population distributions required in parametric tests. The Holm-Bonferroni correction was applied where necessary, to avoid type-I error.

\section{Results}

Velocity traces from each of the three experimental blocks (slow, medium and fast) of one participant are shown in Fig. 2.

The frequencies at which the participants performed the three blocks (slow, medium, and fast), with and without visual feedback, are plotted in Fig. 3. Average movement frequencies were slightly but significantly higher during the vision trials, compared with the blind trials, in the slow and the medium blocks $(p<0.0005)$. Figure 3 demonstrates that participants did not perform the task at the entire range of allowed frequencies, but rather executed the task at a narrow range of frequencies.

Figure 4 is showing accuracy of combined speed and position, measured throughout the entire trial, for the three different blocks, vision and blind, corresponding to the wide, circular and tall ellipses on the phase plane. As we expected, accuracy scores on the vision trials expressed as the percent of the movement time spent inside the target zone on the phase plane, were lower (significantly,

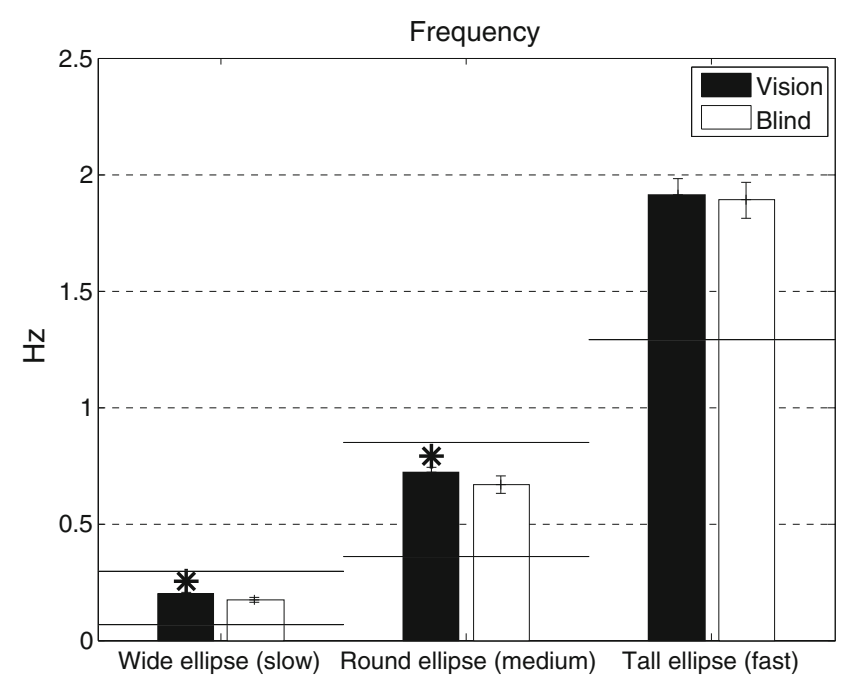

Fig. 3 Frequency values for the vision and no-vision conditions in each of the three blocks (slow, medium, fast). An asterisk denotes a significant difference $(p<0.0005)$ between the vision and the no-vision conditions in the block. The horizontal bars mark the allowed range for each block (only the lower end of the range is shown for the 'fast' block). Error bars represent standard error

$p<0.005)$ when participants performed the medium block compared to the slower block. However, rather than a further drop in accuracy when tracing the tall ellipse on the phase plane, participants' accuracy scores were significantly $(p<0.0125)$ higher in that block compared to the medium one. Aging often affects motor performance in terms of speed as well as accuracy (e.g., Smith et al. 1999; Poston et al. 2008). In order to test whether age plays a role in the observed differences among the blocks, we separated the participant population into two groups, by age: a young group (22-39 years, 10 participants) and an old group (6581 years, 13 participants), and repeated the analysis separately for each group. As expected, the younger group performed better than the older group (see Fig. 5). However, this difference between the groups reached significance only in the intermediate block, and the overall trend was maintained in both groups. Having established that this trend is consistent in both age groups and that in two out of the three blocks performance did not differ significantly between the two groups, we re-pooled the data for further analysis. Using paired statistical analysis ensured that each participant served as his/her own control, as relative, rather than absolute, performance was compared across experimental conditions. Performance in the absence of visual feedback was significantly lower in each of the blocks (22, 48 and $32 \%$ lower than the vision scores in the slow, medium and fast blocks, respectively; $p<0.003)$. In the blind trials, as in the vision trials, a significant drop in accuracy was found between the slow and medium blocks $(p<0.0001)$, and a significant increase between the medium and the fast blocks $(p<0.0005)$. 
Fig. 4 Accuracy (percent time in doughnut) values for the three vision (black) and three blind (white) blocks. Asterisk significant difference between two blocks. Shown here are (1) differences between vision and blind trials within the same block type (slow/medium/fast, $v / b),(2)$ differences among the three vision blocks $(v / v)$, and differences among the three blind blocks $(b / b)$. Error bars represent standard error
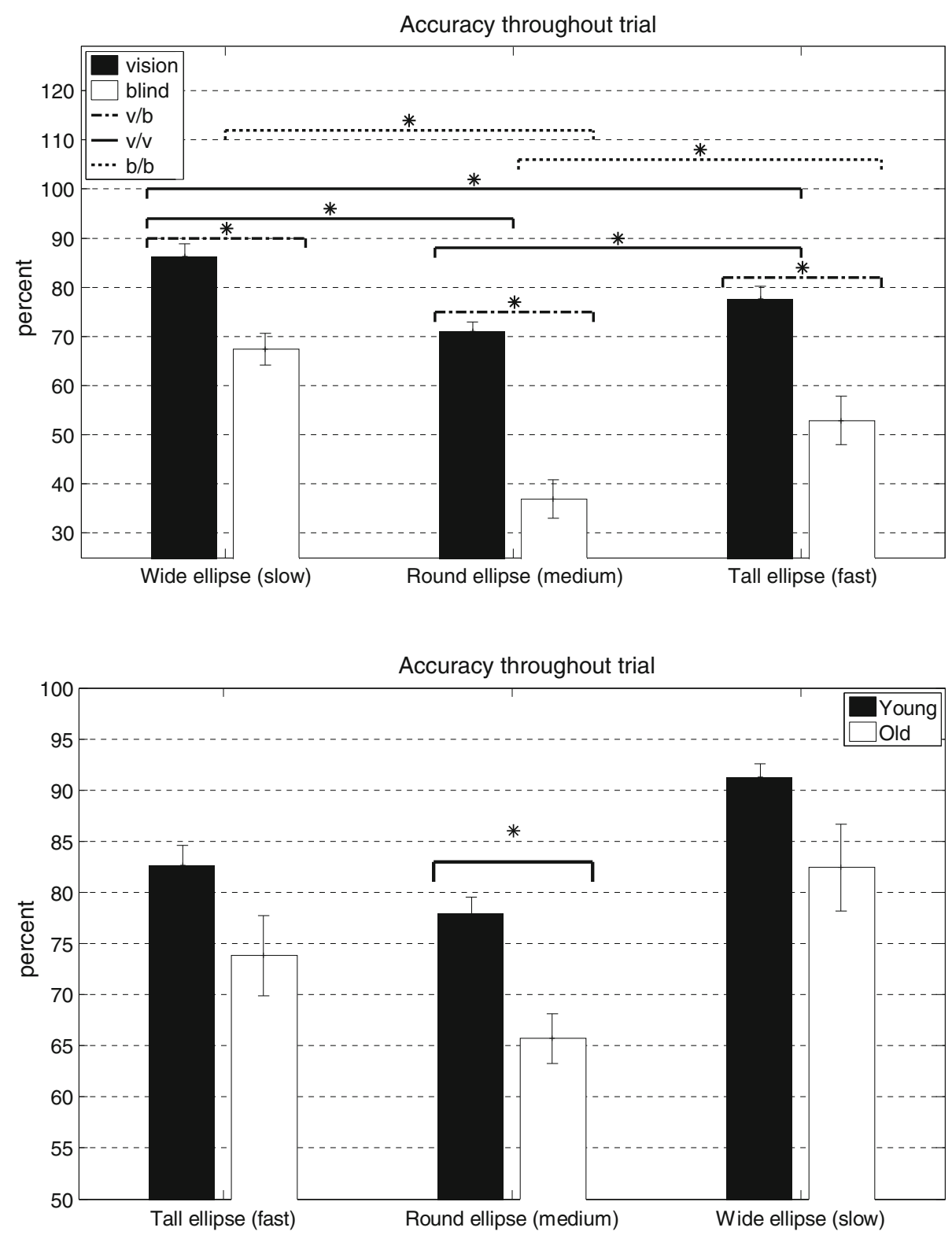

Fig. 5 Accuracy values for the two age groups across the three vision blocks. Asterisk significant difference between the groups $(p<0.05)$. Error bars represent standard error
This non-monotonic relation between the accuracy on the task and the speed at which the task is performed suggests that there may be multiple sources of movement accuracy, and that these become either more or less important depending on the movement speed. We explored the differences among the blocks in an effort to identify potential sources of accuracy.

We analyzed the accuracy data from each quadrant separately (see Fig. 6). A Freidman test was used to examine the effect of quadrant on movement accuracy, followed by a multiple comparison test, where warranted. In the slow block, accuracy values from all four quadrants were similar $(86.2 \pm 2.4-87.2 \pm 3.5 \%)$ and did not differ significantly from one another. In the fast block, a main effect of quadrant on movement accuracy was found $(p<0.025)$. In this block, there is evidence that movements were on average more accurate at movement reversals ( $E$ and $W, 80.3 \pm 3.4$ and $82.1 \pm 2.6 \%$, respectively), than around target peak velocity ( $N$ and $S$, $76.7 \pm 3.1$ and $74.7 \pm 2.7 \%$, respectively), but a significant difference was found only between the $S$ and the $W$ quadrants. In the intermediate block, a main effect of quadrant on movement accuracy was also found $(p<0.0001)$. In this block, the trend observed in the fast block reaches significance. The $E(80.1 \pm 1.7 \%)$ and $W$ $(77.3 \pm 2.2 \%)$ quadrants each are significantly more accurate than the $N(61.5 \pm 3.5 \%)$ and $S(65.1 \pm 2.1 \%)$ quadrants, whereas there is no significant difference between the $E$ and $W$ or between the $N$ and $S$ quadrants.

A Freidman test revealed a significant main effect of quadrant on movement variability $(p<0.0001)$. Post hoc tests showed that the variability in the movement path in all 
Fig. 6 Accuracy values by quadrant. $N$ and $S$ refer to quadrants centered about positive and negative target peak velocity, respectively; $E$ and $W$ refer to quadrants centered about movement reversals: from extension to flexion, and from flexion to extension, respectively. Asterisk significant difference between two quadrants. Error bars represent standard error

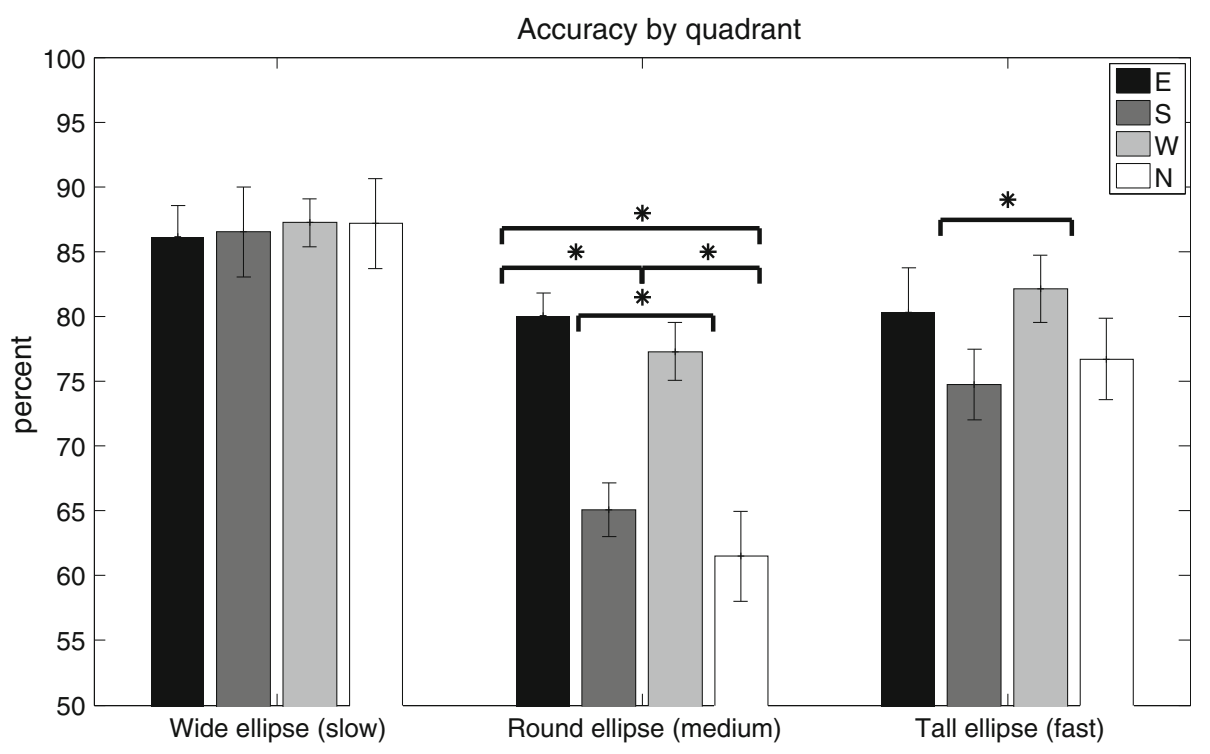

three blocks was significantly higher in the $N$ and $S$ quadrants than in the $E$ and $W$ quadrants (see Fig. 7).

The accelerative phase comprised $39.5 \pm 1.6 \%$ of the half-cycle time in the slow block, and $47.6 \pm 1.0$ and $52.1 \pm 0.02 \%$ in the intermediate and fast blocks, respectively (see Fig. 8). Values for all three blocks were significantly different from one another $(p<0.005)$.

From Fig. 2, it appears that the slower movements are more intermittent than the faster ones. Indeed, using the MSJR measure, we found that smoothness decreased with decreasing movement speed ( $p<0.005$; see Fig. 9). We employed a power-law model to represent the change in MSJR with the average frequency:

MSJR $=a \times$ frequency $^{b}$

For $a=0.02 \mathrm{~s}^{6}$ and $b \approx-6$, this model accounts for $92 \%$ of the variation in the data. It is important to note that an MSJR value close to unity, as found for the fast block $(\mathrm{MSJR}=1.21)$ indicates movement that is nearly maximally smooth. The smoothest back-to-back sequence of discrete movements was found to yield an MSJR value of 6 (Hogan and Sternad 2007); in the medium condition the mean MSJR value was 6.35 , and in the slow condition it approached a value of 600 .

Harmonicity values for the three blocks are shown in Fig. 10. Differences among all blocks were significant $(p<0.0001$; see Fig. 10). Of particular note is that the mean harmonicity value for the fast vision block was 0.95 (0.97 in the blind trials; the difference is significant $p<0.0005$ ), indicating movements of harmonic nature, whereas the mean harmonicity values for the medium and slow blocks were $0.3(0.25, \mathrm{~ns})$ and $0.0008 \mathrm{~ns}(0.004, \mathrm{~ns})$, respectively, supporting an interpretation that they are
Fig. 7 Variability in the phaseplane path by quadrant. $N$ and $S$ refer to quadrants centered about positive and negative target peak velocity, respectively; $E$ and $W$ refer to quadrants centered about movement reversals: from extension to flexion, and from flexion to extension, respectively. Asterisk significant difference between two quadrants. Error bars represent standard error
Variability in phase-plane path

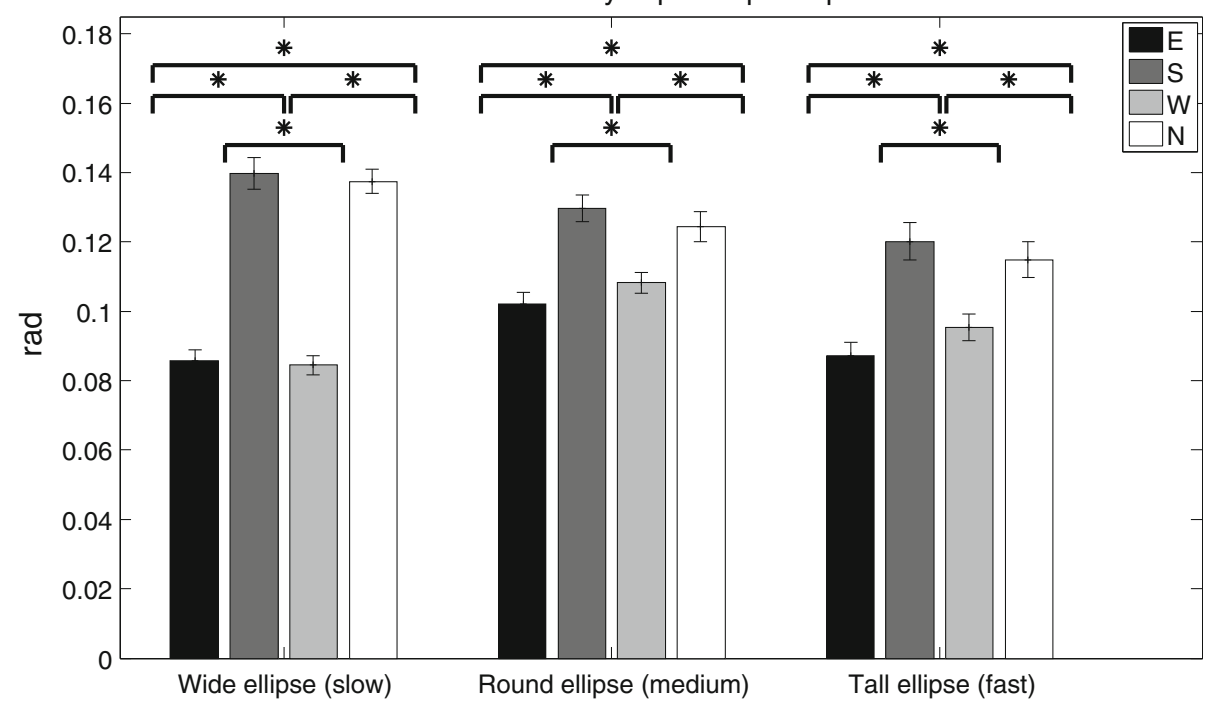


Fig. 8 Acceleration time as a portion of half-cycle time for the three vision blocks. Two vertical asterisks denote this block is significantly different from both others $(p<0.005)$. Error bars represent standard error

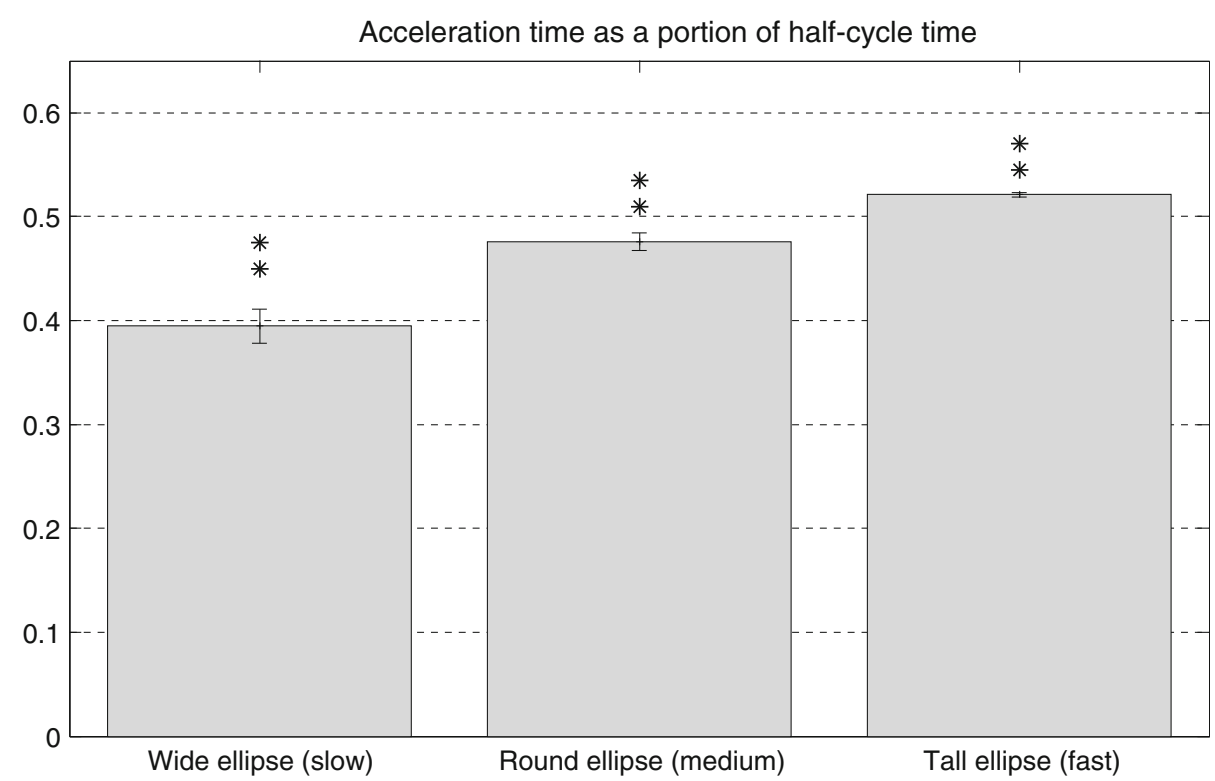

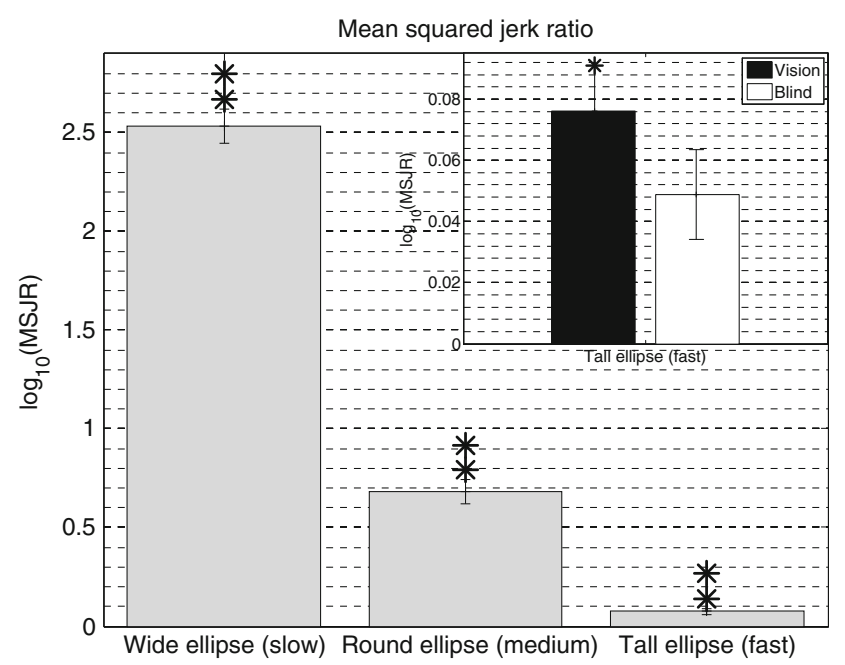

Fig. 9 MSJR (relative smoothness) values for the three vision blocks (slow, medium, fast). The logarithm of the values is displayed, to facilitate comparison among the blocks. Inset fast vision and blind MSJR values. Single asterisk significant difference between the vision and blind trials $(p<0.0005)$; two vertical asterisks denote this block is significantly different from both others $(p<0.005)$. Error bars represent standard error

composed of a string of discrete action units (Guiard 1993; Buchanan et al. 2006; Ketcham et al. 2007).

Postures were found to exist in $99.3 \%$ of movement reversals in the slow vision block, $52.2 \%$ in the intermediate and in $1.0 \%$ of movement reversals in the fast vision block (see Fig. 11). Values were significantly different among the three blocks $(p<0.0001)$. Values for the blind trials were similar to those for the vision trials $(97.9,52.5$ and $0.78 \%$ for the slow, medium and fast blocks, respectively), and did not differ significantly from them.

\section{Discussion}

Summary of results

We found a non-monotonic relation between the speed of movement and the combined accuracy of speed and position. While accuracy scores dropped from the slow to the medium speed, they increased between the medium and the fast speeds. This trend persisted in the data regardless of the age of the participants performing the movements. We explored potential indicators of different movement types in the three experimental blocks, which may point to utilization of alternative sources of movement accuracy. We found that movements in the fast block were nearly maximally smooth, and nearly maximally harmonic. In contrast, movements in the medium and slow blocks were approximately as smooth as the smoothest concatenation of discrete movements, or less, and had mean harmonicity values not greater than 0.3 , supporting an interpretation that they are composed of a string of discrete action units (Buchanan et al. 2006). Furthermore, nearly $100 \%$ of movement reversals in the slow block and more than 50\% of those in the medium block were punctuated by postures, compared with $1 \%$ of movement reversals in the fast block.

These results suggest that when performing movements in the fast block, which were more rhythmic in nature, participants may have used the elastic properties of the limb to achieve not only high smoothness, and to conserve mechanical energy (Guiard 1997; Mottet and Bootsma 1999), but also to achieve repeatability (van Mourik and Beek 2004), and hence accuracy.

In contrast, in the medium and the slow blocks, participants may have made use of visual feedback to achieve movement accuracy, and within these blocks, slower 
Fig. 10 Harmonicity values for the three vision (black) and blind (white) blocks. Asterisk significant difference between two blocks $(p<0.0005)$. Shown here are (1) differences between vision and blind trials within the same block type (slow/medium/fast, $v / b$ ), and (2) differences among the three vision blocks $(v / v)$. Error bars represent standard error
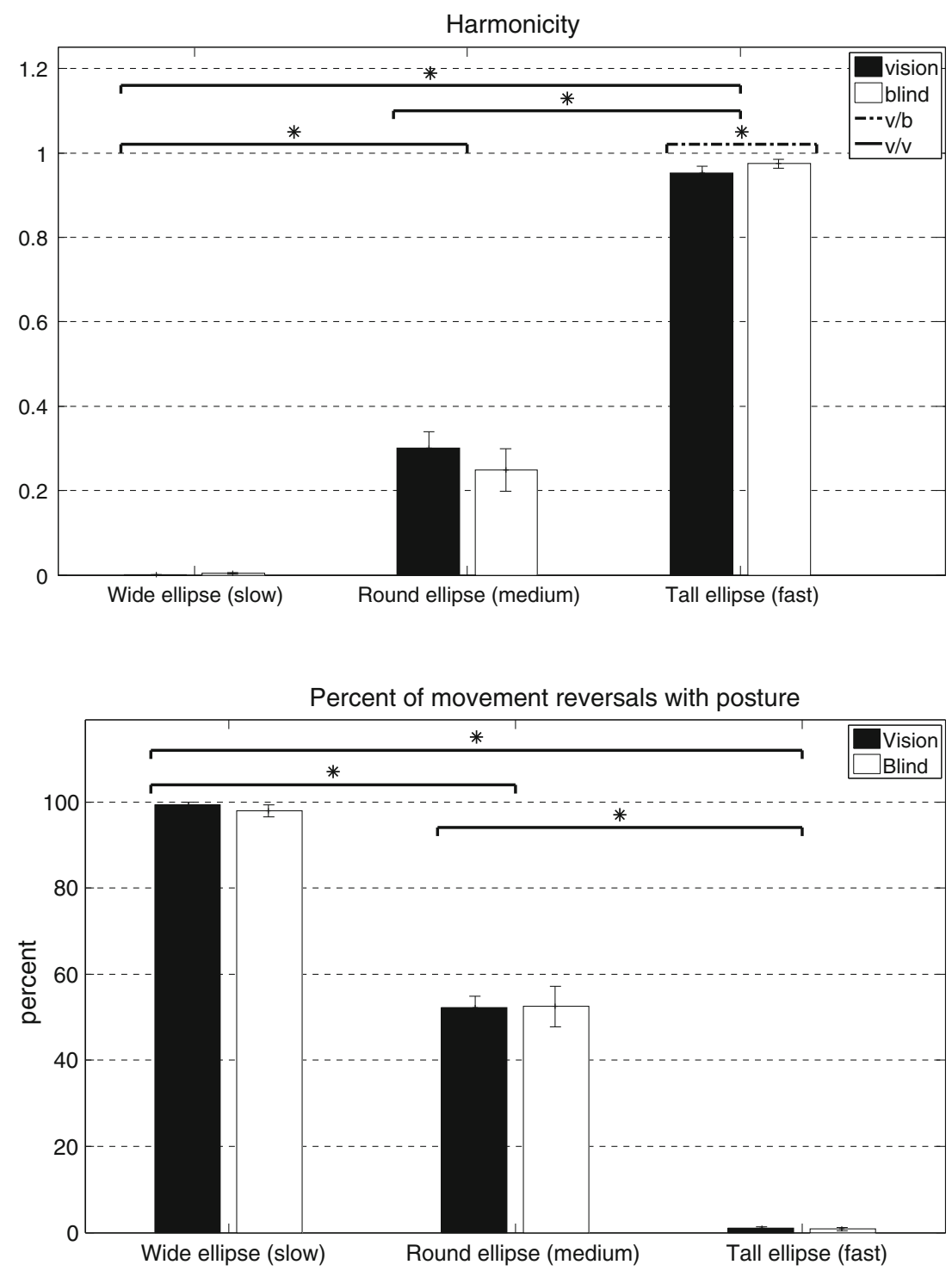

Fig. 11 Percent of trials with postures in the three vision (black) and blind (white) blocks. Asterisk significant difference exists between two vision blocks $(p<0.0001)$. Error bars represent standard error movements afford more time for visual corrections, and are therefore at once more accurate and less smooth.

Indeed, in the slow block the accelerative phase in each half cycle was the shortest of all three blocks, consistent with the interpretation that more time was spent processing visual feedback in each half cycle (Buchanan et al. 2003). This may also be the result of a more flat target velocity profile, indicated by the short, wide ellipse on the phase plane. In the intermediate block the accelerative phase was significantly longer, affording less time for visual feedback processing. Whereas accuracy remained approximately constant throughout the entire cycle in the slow block, in the intermediate block, where less time was available for visual feedback-based corrections, it was significantly lower around \pm target peak velocity (see Fig. 6). While yet less time was available in the fast block for such corrections, average accuracy values around \pm target peak velocity were higher in this block, suggesting a different, non-feedback-based mechanism was employed to achieve accuracy in those trials.

While the task requirements in the intermediate block afforded less time for vision-based corrections than in the slow block, participants in fact relied on that feedback to a larger extent in this block, as evidenced by the larger relative drop in accuracy upon withdrawal of visual feedback in the intermediate block $(\sim 50 \%)$ compared with the slow block $(\sim 20 \%)$. The shape of the target ellipse in the slow block allowed for a relatively wider spread of speeds (in terms of percent of mean target peak speed), thus less susceptible to removal of visual feedback than the medium block. A drop of $30 \%$ in the accuracy of the fast trials upon withdrawal of visual feedback suggests that participants benefit from visual feedback in this block, most likely for calibration of the neutral position, as well as the horizontal and vertical extent, over successive cycles within a trial. 
In the intermediate block, movements were not only less accurate in the $N$ and $S$ quadrants, but also most variable in those quadrants. In fact, these quadrants exhibited a higher variability compared with the $E$ and $W$ quadrants in all three blocks. The different number of cycle repetitions in each block limits the ability to compare variability from block to block. Yet, it is interesting to note that in the $E$ and $W$ quadrants, where path variability is lower, movements in the intermediate block are on average more variable than the corresponding movements in both the fast and the slow blocks. This may indicate that an instability emerges as movements are performed close to the boundary between two types of movements (Buchanan et al. 2006).

The finding that movements performed at the fastest speed required by the experimental paradigm were more accurate than at the intermediate speed, in terms of co-modulation of speed and position throughout the movement, was surprising because we expected the broadly applicable principle that there is a trade off between movement speed and accuracy to apply here as well.

\section{Movement amplitude}

It may be argued that in the fast block participants had the benefit of smaller-amplitude movements, which may have contributed to the increased accuracy. While we believe movement amplitude plays an important role, it is not likely to be the sole reason for the increased accuracy observed in the fast block compared with the medium block. When comparing the slow and the medium blocks, movement accuracy declines in the medium compared with the slow, despite a decrease in amplitude, suggesting that the smaller amplitude in the fast block is not uniquely responsible for the increased accuracy.

\section{Mechanical filtering?}

It may be that "mechanical filtering" due to (1) the inertial properties of the moving limb or (2) the increase in stiffness with speed (Nagasaki 1991; van Galen and de Jong 1995) account for the smoothing of the movement at high frequencies. We demonstrate that this is not the case, as evidenced by a small, yet significant, increase in intermittency with vision in the fast block, compared to the no-vision condition $[1.21 \pm 0.05$ vs. $1.13 \pm 0.04$ (mean \pm SE); see Fig. 9 inset], despite a nonsignificant change in frequency (see Fig. 3). In other words, the smoothness is found to be significantly different between two conditions where the limb moves at essentially indistinguishable frequencies. The observation that movements at the highest frequency were approaching maximal smoothness cannot, therefore, be dismissed as a mere artifact of biomechanics. That does not, however, exclude the possibility that the mechanical properties of the limb were being harnessed to produce a highly smooth movement. This is a subtle, yet important point: while we argue that mechanical filtering is not the reason for high smoothness values at high frequencies, the mechanical properties of the limb are likely exploited, such that all that needs to be set for a movement is the neutral position and muscle stiffness, thereby preprogramming the amplitude and frequency of the movement (Nelson 1983), and intervention is only in the form of an escapement - a mechanism whereby energy is released at exactly the amount needed to compensate for dissipative losses (Kelso et al. 1981) thereby operating as a limit-cycle oscillator.

Information processing versus energy-saving considerations

Many of the theories that were brought forth to describe the reasons for the speed-accuracy dependence, though successful in describing the kinematic aspects of the movement, do not consider the biomechanical muscle properties and their role in controlling endpoint accuracy (cf. van Galen and de Jong 1995; Guiard 1997; Khan and Franks 2000; SmitsEngelsman et al. 2002). Smits-Engelsman et al. (2002, 2006) suggest three of the possible reasons for what they found to be a higher information-processing rate in cyclic than in discrete movements: use of spinal neural oscillators, alternative sources of force, and a more cost-effective use of the recruited force. They cite physiological studies showing that contractions are more effective when they occur in muscles which have just previously been stretched, and when they are eccentric, rather than concentric. Smits-Engelsman et al. (2002) offer an explanation for what may happen at the discrete/rhythmic interface: they argue that possibly, when performing cyclic movements, alternative sources of force recruitment are used, such as the elasticity of muscles and tendons. These may account for a check on the increase in impulse variability and may, in fact, contribute to the observed higher accuracy scores in the fast block.

Furthermore, in a rhythmic movement, the limb need not come to a full rest at the position extremes, such that acceleration does not equal zero at those points. Energy is saved in eliminating the need to coordinate agonist and antagonist muscle activity to reach a full stop before initiating the next movement portion (Guiard 1997). That maximally smooth movement is within $2 \%$ of that which minimizes energy expenditure has been demonstrated mathematically (Nelson 1983). Energy-saving considerations have been brought up in the context of gait patterns-e.g., running versus walking (Alexander 1991) and choice of gait type has been discussed in terms of both speed and step amplitude (Srinivasan and Ruina 2006), and these may well be paralleled in the upper limbs. 
Discrete versus rhythmic movements: can it explain our findings?

Our salient finding that at the highest examined movement speed (the fast block), as well as at the largest examined movement amplitude (the slow block), accuracy was higher than at an intermediate speed and amplitude (the medium block) seems at odds with the theories put forth by Schmidt et al. (1979): they posited that, for discrete rapid aiming movements, increased speed necessitates increased impulse size, which, in turn, leads to an increase in output variability; for reciprocal movements, they asserted that output variability is proportional to movement amplitude only. However, on the reciprocal task at hand, we found movements at larger amplitudes to be more accurate. What may account for this finding? It has been suggested that continuous cyclic arm movements, if performed sufficiently slowly, exhibit kinematic features that suggest they may be executed as a sequence of discrete movements (Buchanan et al. 2006; Hogan and Sternad 2007), which may be described as overlapping movement units (Morasso and Mussa Ivaldi 1982; Flash and Henis 1991; Krebs et al. 1999). If, as we argue, the movements in the slow and the medium blocks in our experiment fall under this definition - that is, if they are executed as a string of discrete movements, as is suggested by the smoothness and harmonicity analyses - then Schmidt et al.'s (1979) theory regarding discrete movements holds for these two blocks. Namely, the faster speed in the intermediate condition compared to the slow condition resulted in increased error. That is, if we consider the slow and the medium blocks in isolation from the fast block, then the model suggested by Schmidt et al. (1979) correctly predicts the decrease in accuracy with an increase in speed.

Can the movements in the slow and the medium blocks be classified as more discrete in nature and ones in the fast block as more rhythmic in nature?

Indeed, a remarkable parallel can be observed between the fast $(\sim 2 \mathrm{~Hz})$ movements in our experiment and maximally smooth movements on the one hand; and between the movements in the medium $(\sim 0.6 \mathrm{~Hz})$ and slow $(\sim 0.2 \mathrm{~Hz})$ ranges in our experiment and a concatenation of discrete movements on the other (Doeringer and Hogan 1998). The former are fast, yet relatively accurate and smooth, while the latter exhibit a decrease in accuracy and increase in smoothness with increasing speed. This is despite the fact that the task instructions were uniform throughout the experiment, calling for cyclic movements only (none of the tasks was discrete in nature).

Schaal et al. (2004), using functional neuroimaging, found that rhythmic movement activated a small number of unilateral primary motor areas, whereas discrete movement activated additional contralateral nonprimary motor areas and showed a strong bilateral activity in the cerebrum and the cerebellum. These researchers studied movements that belonged unambiguously to one of the two categories (discrete and rhythmic). The results of the current study, together with results from Buchanan et al. (2006), suggest the task conditions under which participants may choose to switch from employing one type of movement to another, thereby allowing a directed exploration of the brain-activation pattern associated with the "grey area" between distinctly continuous and distinctly discrete movement generation.

\section{Two regimes}

These findings suggest a possible separation of the frequency/amplitude plane of repetitive movements into what may be called a "truly cyclic" regime, at frequency values in the vicinity of the inverse of the visual reaction time $(>1 \mathrm{~Hz})$, and small amplitudes, where movements approach maximal smoothness, and a "pseudo-cyclic" regime at lower frequencies and higher amplitudes, where movements are not maximally smooth, and higher speed results in decreased accuracy and increased smoothness. Movements in both regimes are rhythmic in the sense that no planned stop is made between half cycles, and movement is performed continuously. Any postures, as defined quantitatively above, are the result of the very slow required movement, and are likely a manifestation of the motor system's inability to perform movements that are that slow in a smooth manner (Doeringer and Hogan 1998). However, movements in the "pseudo-cyclic" regime share many features with discrete movements. In our experiment, amplitude and frequency were co-varied, and therefore, we cannot comment on their individual contributions to the choice of regime. Additionally, within what we term the "truly cyclic" regime, accuracy and smoothness may vary with speed, but we cannot comment on the form of the function, since we only measured behavior on a single frequency in that regime. Nagasaki's results (1991) suggest a third regime, above $3-4.3 \mathrm{~Hz}$, where movements cease to be "rhythmic" and adopt a symmetric non-linear control mechanism. The boundaries that define these regimes may well depend on the executing limb and/or the limb segment.

Plamondon and Alimi (1997) suggested a model for rapid-aimed movements in which intermittency is not the result of feedback-based "corrections", but is part of a welltrained feedforward loop, which echoes suggestions made by Meyer et al. (1988) and Elliott et al. (2001). Intermittency, then, may be, at least in part, the result of limitations on the frequency/amplitude of the basic submovement. That is, there may be limits on the duration and amplitude of what may constitute a single, uninterrupted smooth motion. 
Movements that take longer to complete, or that stretch over larger amplitudes may necessitate the concatenation of several such movement subunits. As such, these movements would be characterized by high intermittency, as the jerk of a reciprocal maximally smooth movement is $1 / 6$ th of that of the smoothest back-to-back sequence of discrete movements (Hogan and Sternad 2007).

At the high-frequency end of the "pseudo-cyclic" regime, movements are inherently less smooth than maximally smooth movements, unable to benefit from the biomechanically induced increased accuracy privileges of the "truly cyclic" regime and, at the same time, have more limited time than lower-frequency movements to make use of feedback during the movement. Such a mechanism is congruent with our findings that show smoothness to decrease monotonically with decreasing speed, and that reveal movements in the intermediate block to have the lowest accuracy scores of all three examined frequencies.

It is important to keep in mind that the two "regimes" that we describe are not the result of different experimental procedures. Rather, the results are all the more illuminating because the type of task required of the participants was identical: they were asked to perform a rhythmic motion in all three conditions. That they produced a maximally smooth movement in one of the conditions (fast, smallamplitude movements), and more intermittent movements in the two other conditions (slower, larger-amplitude movements), and that the accuracy score was not a monotonic function of speed suggests that when crossing a frequency/amplitude boundary, participants switch to a different, possibly more energy-efficient mode, characterized by maximal smoothness and relatively higher accuracy. A similar notion of a frequency-dependence of the control mechanisms underlying cyclic motions was advanced by Nagasaki (1991), though he investigated transitions at higher frequencies. He also noted a decrease in energy expenditure when participants moved at frequencies greater than $3.3 \mathrm{~Hz}$.

\section{Conclusions}

In summary, we tested participants on a task involving rhythmic forearm movements at three speeds. We found that movement smoothness decreased with decreasing speed. However, accuracy of speed and position measured throughout the trajectory was not a monotonic function of speed. Movements at the intermediate condition were significantly less accurate than movements performed at either a higher or a lower speed. We suggest a model of rhythmic movements where the plane of the movements' frequency/ amplitude combinations is separated into two regimes. In the high-frequency, small-amplitude regime, movements approach maximal smoothness, possibly due to an exploitation of the elastic properties of the limb for achievement of movement accuracy. In the low-frequency, largeamplitude regime, movements are not maximally smooth, possibly due to the increased required duration and distance, and are more intermittent and more accurate with decreasing frequency, probably due to the dependence on sensory feedback for achievement of movement accuracy. The results of this study warrant further investigation into the individual contributions of movement duration and movement amplitude to movement smoothness.

Acknowledgments This work was supported in part by the National Institute of Child Health and Human Development/National Center for Medical Rehabilitation Research (NICHD/NCMRR) grant 1 R01HD045343 and NIH grant 2 R01-NS036449; and the VA Veterans Affairs grants B3688R and B3607R. S. Levy-Tzedek was supported in part by a Howard Hughes Medical Institute Predoctoral Fellowship and by the VA Veterans Affairs grants B3688R and B3607R at MIT, and is currently funded by a Kreitman Foundation Postdoctoral Fellowship at the Ben Gurion University.

Open Access This article is distributed under the terms of the Creative Commons Attribution Noncommercial License which permits any noncommercial use, distribution, and reproduction in any medium, provided the original author(s) and source are credited.

\section{References}

Alexander RM (1991) Energy-saving mechanisms in walking and running. 160:55-69

Beamish D, Bhatti SA, MacKenzie IS, Wu J (2006) Fifty years later: a neurodynamic explanation of Fitts' law. J R Soc Interface 3:649 654

Bootsma RJ, Fernandez L, Mottet D (2004) Behind Fitts' law: kinematic patterns in goal-directed movements. Int $\mathrm{J}$ Hum Comput Stud 61:811-821

Buchanan JJ, Park JH, Ryu YU, Shea CH (2003) Discrete and cyclical units of action in a mixed target pair aiming task. Exp Brain Res 150:473-489

Buchanan JJ, Park JH, Shea CH (2004) Systematic scaling of target width: dynamics, planning, and feedback. Neurosci Lett 367:317-322

Buchanan JJ, Park JH, Shea CH (2006) Target width scaling in a repetitive aiming task: switching between cyclical and discrete units of action. Exp Brain Res 175:710-725

Crossman ER, Goodeve PJ (1983) Feedback control of handmovement and Fitts' Law. Q J Exp Psychol A 35:251-278

Doeringer JA, Hogan N (1998) Intermittency in preplanned elbow movements persists in the absence of visual feedback. J Neurophysiol 80:1787-1799

Elliott D, Helsen WF, Chua R (2001) A century later: Woodworth's (1899) two-component model of goal-directed aiming. Psychol Bull 127:342-357

Fitts PM (1954) The information capacity of the human motor system in controlling the amplitude of movement. J Exp Psychol 47:381-391

Fitts PM, Peterson JR (1964) Information capacity of discrete motor responses. J Exp Psychol 67:103-112

Flash T, Henis E (1991) Arm trajectory modifications during reaching towards visual targets. J Cogn Neurosci 3:220-230 
Guiard Y (1993) On Fitts's and Hooke's laws: simple harmonic movement in upper-limb cyclical aiming. Acta Psychol (Amst) 82:139-159

Guiard Y (1997) Fitts' law in the discrete vs. cyclical paradigm. Hum Mov Sci 16:97-131

Hogan N, Sternad D (2007) On rhythmic and discrete movements: reflections, definitions and implications for motor control. Exp Brain Res 181:13-30

Kelso JA, Tuller B (1984) Converging evidence in support of common dynamical principles for speech and movement coordination. Am J Physiol Regul Integr Comp Physiol 246:928-935

Kelso JA, Southard DL, Goodman D (1979) On the nature of human interlimb coordination. Science 203:1029

Kelso JA, Holt KG, Rubin P, Kugler PN (1981) Patterns of human interlimb coordination emerge from the properties of non-linear, limit cycle oscillatory processes: theory and data. J Mot Behav 13:226-261

Ketcham CJ, Rodriguez TM, Zihlman KA (2007) Targeted aiming movements are compromised in nonaffected limb of persons with stroke. Neurorehabil Neural Repair 21:388

Khan MA, Franks IM (2000) The effect of practice on component submovements is dependent on the availability of visual feedback. J Mot Behav 32:227-240

Krebs HI, Aisen ML, Volpe BT, Hogan N (1999) Quantization of continuous arm movements in humans with brain injury. Proc Natl Acad Sci USA 96:4645-4649

Meyer DE, Abrams RA, Kornblum S, Wright CE, Smith JE (1988) Optimality in human motor performance: ideal control of rapid aimed movements. Psychol Rev 95:340-370

Morasso P, Mussa Ivaldi FA (1982) Trajectory formation and handwriting: a computational model. Biol Cybern 45:131-142

Mottet D, Bootsma RJ (1999) The dynamics of goal-directed rhythmical aiming. Biol Cybern 80:235-245

Nagasaki H (1991) Asymmetrical trajectory formation in cyclic forearm movements in man. Exp Brain Res 87:653-661

Nelson WL (1983) Physical principles for economies of skilled movements. Biol Cybern 46:135-147
Plamondon R, Alimi AM (1997) Speed/accuracy trade-offs in targetdirected movements. Behav Brain Sci 20:279-303 (discussion 303-249)

Poston B, Enoka JA, Enoka RM (2008) Endpoint accuracy for a small and a large hand muscle in young and old adults during rapid, goal-directed isometric contractions. Exp Brain Res 187:373385

Ricker KL, Elliott D, Lyons J, Gauldie D, Chua R, Byblow W (1999) The utilization of visual information in the control of rapid sequential aiming movements. Acta Psychol 103:103-123

Schaal S, Sternad D, Osu R, Kawato M (2004) Rhythmic arm movement is not discrete. Nat Neurosci 7:1136-1143

Schmidt RA, Zelaznik H, Hawkins B, Frank JS, Quinn JT Jr (1979) Motor-output variability: a theory for the accuracy of rapid motor acts. Psychol Rev 47:415-451

Smith CD, Umberger GH, Manning EL, Slevin JT, Wekstein DR, Schmitt FA, Markesbery WR, Zhang Z, Gerhardt GA, Kryscio RJ, Gash DM (1999) Critical decline in fine motor hand movements in human aging. Neurology 53:1458-1461

Smits-Engelsman BC, Van Galen GP, Duysens J (2002) The breakdown of Fitts' law in rapid, reciprocal aiming movements. Exp Brain Res 145:222-230

Smits-Engelsman BC, Swinnen SP, Duysens J (2006) The advantage of cyclic over discrete movements remains evident following changes in load and amplitude. Neurosci Lett 396:28-32

Srinivasan M, Ruina A (2006) Computer optimization of a minimal biped model discovers walking and running. Nature 439:72-75

van Galen GP, de Jong WP (1995) Fitts' law as the outcome of a dynamic noise filtering model of motor control. Hum Mov Sci 14:539-571

van Mourik AM, Beek PJ (2004) Discrete and cyclical movements: unified dynamics or separate control? Acta Psychol 117:121-138

Wallace SA, Newell KM (1983) Visual control of discrete aiming movements. Q J Exp Psychol A 35:311-321

Wright CE, Meyer DE (1983) Conditions for a linear speed-accuracy trade-off in aimed movements. Q J Exp Psychol A 35:279-296 